\title{
Modelado y Diseño del Controlador para un Sistema de Tracción de un Vehículo Eléctrico
}

\author{
Miguel A. Durán, Jorge Gudiño, Saida M. Charre y Janeth A. Alcalá \\ Facultad de Ingeniería Electromecánica, Universidad de Colima, km. 20 carretera Manzanillo - Cihuatlán, \\ Manzanillo, Colima- México (e-mail: mduran@ucol.mx, jglau@ucol.mx, scharre@ucol.mx, \\ janethalcala@ucol.mx)
}

Recibido Mar. 5, 2014; Aceptado Jun. 16, 2014; Versión final recibida Ago. 12, 2014

\section{Resumen}

En este artículo se presenta la estructura básica y el diseño de un controlador de par para un vehículo eléctrico impulsado por un motor de inducción. En primer lugar, se presenta la estructura básica del vehículo eléctrico en cuestión y su modelo matemático. Posteriormente, se analiza el principio de funcionamiento del control directo de par convencional y a partir de este análisis se diseña un nuevo controlador con una frecuencia de conmutación constante. Para generar los pulsos de conmutación para el inversor se emplea PWM senoidal en lugar de los comparadores de histéresis y la tabla de conmutación comúnmente utilizados en el control directo de par. Finalmente, se realiza una comparación mediante simulación. Esto muestra el mejor desempeño de la estrategia de control propuesta en este trabajo al presentar menores errores de par y flujo, y menor rizo en el par y en las corrientes del estator.

Palabras clave: vehículo eléctrico, control directo de par, motor de inducción, modelado matemático, simulación

\section{Modeling and Controller Design for a Traction System of Electric Vehicle}

\begin{abstract}
In this paper the basic structure and design of a torque controller for an electric vehicle driven by induction motor is presented. The basic structure of the electric vehicle in question and its mathematical model is presented first. After that, the operation principle of the conventional direct torque control is analyzed and from this a new controller with fixed constant switching frequency is designed. To generate the switching pulses sine PWM is used instead of the hysteresis comparator and switching table commonly used in direct torque control. Finally, simulation comparison was performed. This shows that better performance was achieved with the proposed controller with lower torque and flux errors, and lower torque and stator current ripple.
\end{abstract}

Keywords: electric vehicle, direct torque control, induction motor, mathematical model, simulation 


\section{INTRODUCCIÓN}

Los vehículos eléctricos (VE) tienen varias ventajas sobre los vehículos convencionales que usan normalmente un motor de combustión interna. Dichas ventajas son que pueden recuperar energía cinética en el frenado y energía potencial cuando se recorren caminos en bajada. Esto ha hecho a los VE una buena opción para reducir los efectos negativos asociados a la transportación humana, como lo son la contaminación, el calentamiento global y la reducción en la disponibilidad de los combustibles fósiles, juntos a las consecuencias socio-económicas asociadas a estos efectos (Andrada et al, 2005), (Sciarretta y Guzzella, 2007), (Schouten et al, 2002) y (Gonder, 2008).

Una de las técnicas de control que presentan mejores resultados para el control de motores eléctricos de corriente alterna es el control directo de par (CDP), el cual fue introducido hace más de 25 años por Takahashi (Takahashi y Noguchi, 1986) en Japón y por Depenbrock (Depenbrock, 1985) en Alemania. Esta estrategia de control se utiliza ampliamente en VE (Hegazy et al, 2013). En el CDP convencional se utiliza una tabla para determinar las conmutaciones del inversor (Ahmed et al, 2014). Esta técnica de conmutación es muy simple y fácil de implementar, sin embargo tiene el inconveniente de que la frecuencia de conmutación no es constante (Idris et al, 2006). De acuerdo con (Vasudevan y Arumugam, 2004) las ventajas del CDP son: i) rápida respuesta del par, ii) relativamente simple y iii) no requiere de sensores de velocidad ni posición; y sus desventajas son: i) alta distorsión de corriente, lo que aumenta las pérdidas, ii) rizo de par elevado y iii) frecuencia de conmutación variable. Con el objetivo de evitar estas desventajas, en este trabajo se realiza el diseño de un controlador con las siguientes características: i) frecuencia de conmutación fija utilizando PWM senoidal; ii) reducción de la distorsión en las corrientes del estator; y iii) menor rizo del par generado por el motor. Con esto, el controlador propuesto tiene un mejor desempeño que el CDP convencional utilizado comercialmente. La modulación PWM senoidal es una de las técnicas de conmutación más simples pero efectiva para inversores con fuente de voltaje, debido a que proporciona: un amplio rango de control lineal, rápida respuesta e implementación digital sencilla (Hassankhan y Khaburi, 2008).

\section{MODELADO DEL VEHÍCULO ELÉCTRICO}

En (Durán et al, 2010) se desarrolla el modelo matemático del VE cuyos principales componentes (convertidor CD-CA, motor de inducción trifásico, parte mecánica del VE), se muestran en la figura 1.

Convertidor $C D-C A$ (inversor). Se considera un convertidor CD-CA (corriente directa - corriente alterna), también llamado inversor, como el mostrado en la figura 2. El fasor espacial del voltaje de salida del inversor $\bar{u}_{s}$ depende del voltaje de CD del banco de baterías $V_{c d}$ y de las señales de modulación $S_{a}, S_{b}$ y $S_{c}$, como se expresa en la siguiente ecuación:

$\bar{u}_{s}=\frac{2}{3} V_{c d}\left[1 S_{a}+a S_{b}+a^{2} S_{c}\right]$

Donde:

$a=e^{j 2 \pi / 3}$ y $a^{2}=e^{j 4 \pi / 3}$.

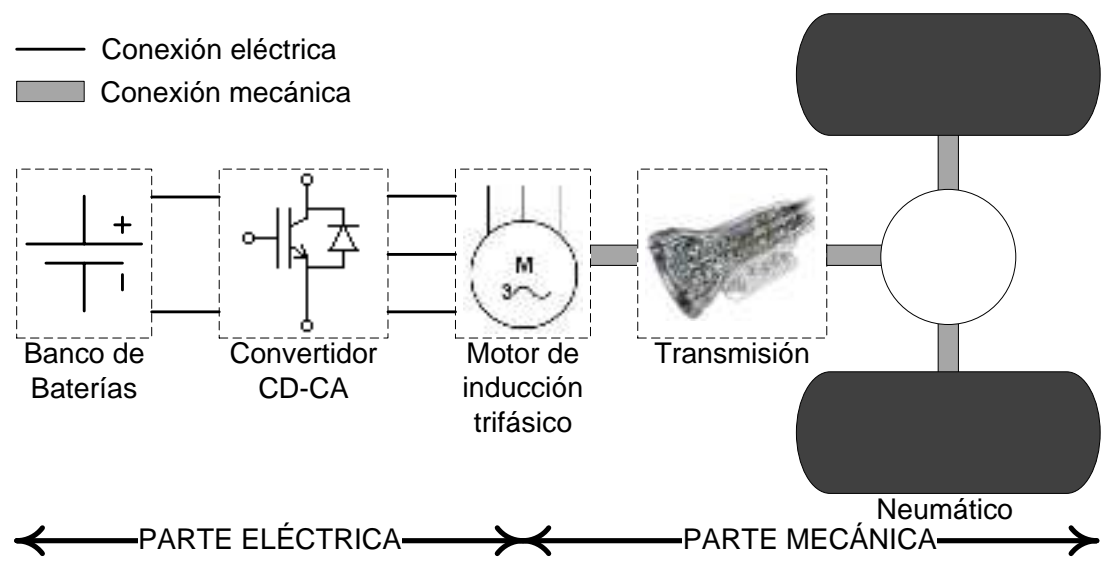

Fig. 1. Diagrama del vehículo eléctrico.

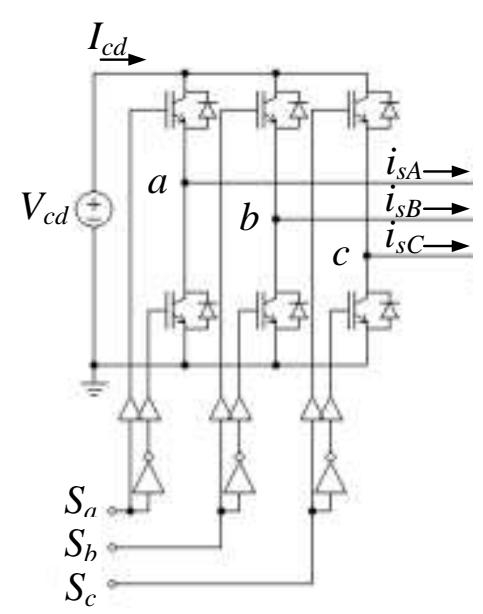

Fig. 2. Convertidor CD - CA. 
Motor de inducción trifásico. El Ml trifásico tipo jaula de ardilla cuenta con un devanado trifásico en el estator, y en el rotor cuenta con una jaula de ardilla que, para propósitos de análisis, se puede considerar como un devanado trifásico con voltaje de alimentación igual a cero. En el marco de referencia (MR) fijo al estator, se tienen dos ecuaciones de voltajes de Kirchhoff (estator y rotor).

$$
\begin{gathered}
\bar{u}_{s}=R_{s} \bar{l}_{s}+\frac{d \bar{\psi}_{s}}{d t} \\
0=R_{r} \bar{\iota}_{r}{ }^{\prime}+\frac{d \bar{\psi}_{r}{ }^{\prime}}{d t}-j \omega_{r} \bar{\psi}_{r}{ }^{\prime}
\end{gathered}
$$

En estas ecuaciones, $\bar{u}_{s}$ es el fasor espacial de voltaje del estator, $\bar{l}_{s}$ es el fasor espacial de corriente del estator, $\bar{\psi}_{s}$ es el fasor espacial de enlaces de flujo del estator, $R_{s}$ es la resistencia de los devanados del estator, $\bar{l}_{r}{ }^{\prime}$ es el fasor espacial de corriente del rotor en el MR estacionario, $\bar{\psi}_{r}{ }^{\prime}$ es el fasor espacial de enlaces de flujo del rotor en el MR estacionario y $R_{r}$ es la resistencia de los devanados del rotor.

El par electromagnético generado por el MI está dado por:

$t_{e}=\frac{3}{2} P \frac{L_{m}}{L_{s}{ }^{\prime} L_{r}} \bar{\psi}_{r}{ }^{\prime} \times \bar{\psi}_{s}$

donde $L_{s}{ }^{\prime}=L_{s}-L_{m}{ }^{2} / L_{r}$, $L_{s}$ es la inductancia total del estator, $L_{r}$ es la inductancia total del rotor, y $L_{m}$ es la inductancia de magnetización.

Parte mecánica del VE. Para el caso de un VE se puede considerar como carga mecánica del motor eléctrico al propio VE. La parte mecánica del VE se entenderá como el conjunto formado por: la transmisión, las ruedas, y la masa total de sus componentes (baterías, convertidores electrónicos, máquina eléctrica, transmisión, carrocería, chasis, etc.).

El modelo de la parte mecánica del VE está dado por:

$$
\begin{aligned}
& \frac{d \omega_{r}}{d t}=\frac{\eta_{g} G^{2}}{J \eta_{g} G^{2}+m r^{2}}\left(t_{e}-B \omega_{r}-\frac{\rho A C_{d} r^{3}}{2 \eta_{g} G^{3}} \omega_{r}^{2}-\frac{\mu_{r r} m g r \cos (\gamma) \operatorname{sign}\left(\omega_{r}\right)}{\eta_{g} G}-\frac{m g r \operatorname{sen}(\gamma)}{\eta_{g} G}\right) \\
& \frac{d \omega_{r}}{d t}=\frac{G^{2}}{J G^{2}+m \eta_{g} r^{2}}\left(t_{e}-B \omega_{r}-\frac{\rho A \eta_{g} C_{d} r^{3}}{2 G^{3}} \omega_{r}{ }^{2}-\frac{\mu_{r r} m g \eta_{g} r \cos (\gamma) \operatorname{sign}\left(\omega_{r}\right)}{G}-\frac{m g \eta_{g} r \operatorname{sen}(\gamma)}{G}\right)
\end{aligned}
$$

La ecuación (4) se utiliza cuando la máquina eléctrica entrega potencia mecánica, es decir, funciona como motor (condiciones normales de operación) y la ecuación (5) se utiliza cuando la máquina eléctrica recibe potencia mecánica, es decir, funciona como generador (frenado regenerativo). $r$ es el radio del neumático, $\eta_{g}$ es la eficiencia de la transmisión, $G$ es el cociente de reducción de velocidad angular, $t_{e}$ es el par proporcionado por el motor eléctrico, $\mu_{r r}$ es el coeficiente de fricción, $m$ es la masa total del VE, $g$ es la aceleración debida a la gravedad, $\operatorname{sign}(\cdot)$ es la función signo, $\rho$ es la densidad del aire, $A$ es el área frontal del VE, $C_{d}$ es el coeficiente aerodinámico, $J$ es la inercia total (rotor y VE), $B$ es el coeficiente de fricción viscosa del Ml y $\gamma$ es el ángulo de la pendiente sobre la que se mueve el VE. Así el modelo del VE está dado por las ecuaciones (1), (2) para la parte eléctrica, (3) para el par desarrollado por el motor y (4), (5) para la parte mecánica.

\section{CONTROLADOR}

CDP convencional: Para entender el CDP se parte de la ecuación (3) del par electromagnético, expresando el par en función de los módulos $\left|\bar{\psi}_{s}\right|$ y $\left|\bar{\psi}_{r}{ }^{\prime}\right|$ y de los ángulos $\rho_{s}$ y $\rho_{r}{ }^{\prime}$, de los fasores espaciales de enlaces de flujo del estator y rotor, respectivamente.

$t_{e}=\frac{3}{2} P \frac{L_{m}}{L_{s}{ }^{\prime} L_{r}}\left|\bar{\psi}_{r}{ }^{\prime}\right|\left|\bar{\psi}_{s}\right| \operatorname{sen}\left(\rho_{s}-\rho_{r}{ }^{\prime}\right)$

Debido a que la constante de tiempo del rotor de un MI tipo jaula de ardilla es grande (Vas, 1998), el fasor espacial de enlaces de flujo del rotor cambia lentamente y puede asumirse constante durante pequeños lapsos de tiempo. Por tanto el control del par se puede llevar a cabo mediante el fasor espacial de enlaces de flujo del estator. A partir de (6) se puede observar que, si se mantiene constantes $\left|\bar{\psi}_{s}\right|$ y $\left|\bar{\psi}_{r}{ }^{\prime}\right|$, el par puede cambiarse rápidamente mediante $\rho_{s}-\rho_{r}{ }^{\prime}$. Por tanto, en el CDP el módulo $\left|\bar{\psi}_{s}\right|$ controla la magnitud 
del flujo y el ángulo $\rho_{s}$ controla el par. Por otra parte, el fasor espacial de los enlaces de flujo del estator (módulo y ángulo) se puede cambiar mediante el fasor espacial de voltaje del estator. Esto se puede ver a partir de la ecuación de voltajes del estator del MI,

$\bar{u}_{s}=R_{s} \bar{l}_{s}+\frac{d \bar{\psi}_{s}}{d t}$

Un inversor de dos niveles (ver figura 2) puede proporcionar seis fasores espaciales de voltaje activos y dos fasores espaciales de voltaje cero, dados por:

$\bar{u}_{k}=\left\{\begin{array}{cc}\frac{2}{3} V_{c d} e^{j[(k-1) \pi / 3]} & k=1,2,3,4,5,6 \\ 0 & k=0,7\end{array}\right.$

Los fasores de voltaje del inversor se muestran en la figura 3. Los números entre paréntesis representan las señales de modulación del inversor $S_{a}, S_{b}$ y $S_{c}$ para cada fasor espacial de voltaje. También se muestra los seis sectores de $\pi / 3$ rad en los que se divide el plano para el CDP convencional. En la figura 4 se muestra un diagrama a bloques del CDP convencional. EI CDP utiliza dos comparadores de histéresis: uno de dos niveles para el error de flujo (figura 5.a) y uno de tres niveles para el error de par (figura 5.b). Las bandas de tolerancia $\Delta \psi$ y $\Delta t_{e}$ establecen los valores permisibles de error para el flujo y el par, respectivamente. De acuerdo a la salida de los comparadores de flujo $\mathrm{d} \psi$ y par $\mathrm{d} t_{e}$, y al sector en la cual se encuentre el fasor espacial de enlaces de flujo del estator, es el valor que se envía a las señales de modulación del inversor de acuerdo con la tabla 1.

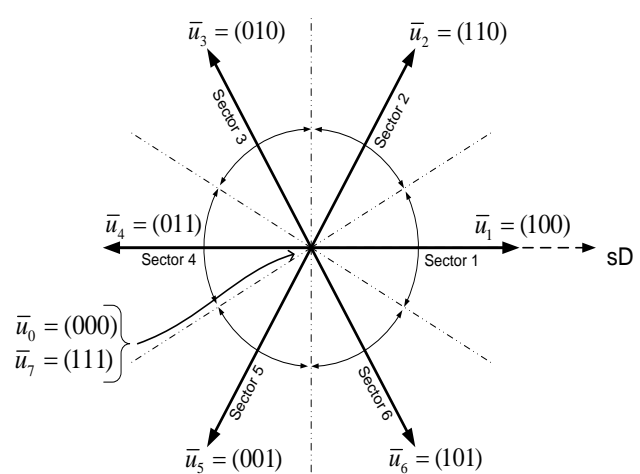

Fig. 3. Fasores espaciales de voltaje del inversor.

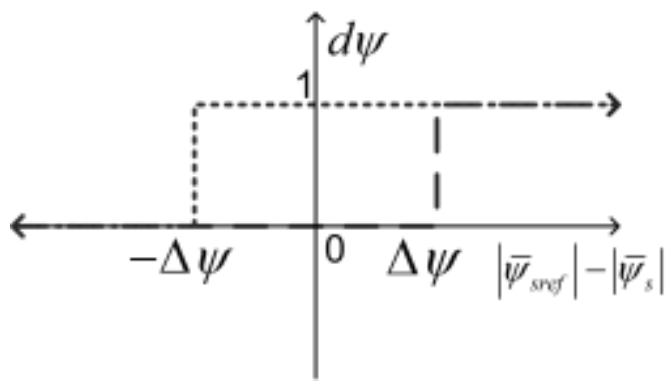

a)

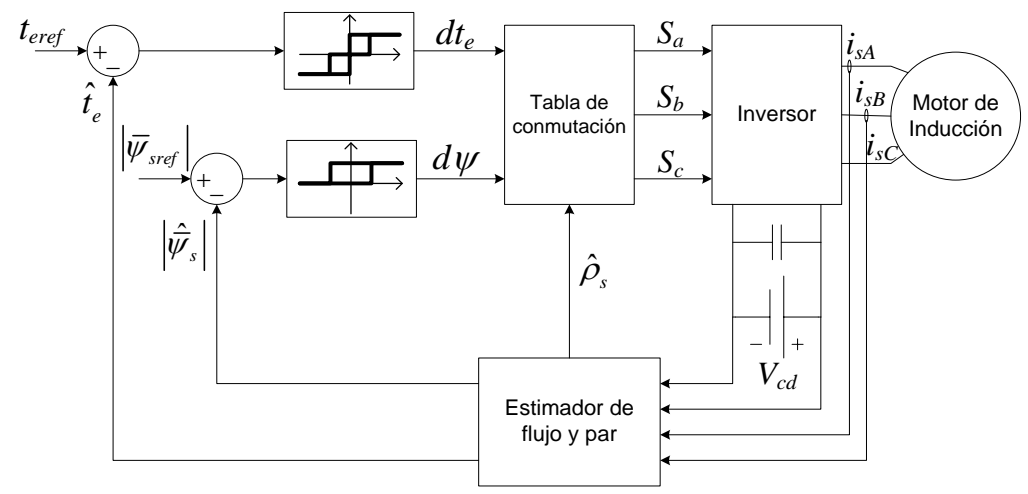

Fig. 4. Diagrama a bloques del CDP convencional.

Fig. 5. Comparadores de histéresis de: a) flujo y b) par

Tabla 1: Tabla de conmutación

\begin{tabular}{|l|l|l|l|l|l|l|l|}
\hline$d \psi$ & $d t_{e}$ & Sector 1 & Sector 2 & Sector 3 & Sector 4 & Sector 5 & Sector 6 \\
\hline \multirow{3}{*}{1} & 1 & 110 & 010 & 011 & 001 & 101 & 100 \\
\cline { 2 - 8 } & 0 & 111 & 000 & 111 & 000 & 111 & 000 \\
\cline { 2 - 8 } & -1 & 101 & 100 & 110 & 010 & 011 & 001 \\
\hline \multirow{3}{*}{0} & 1 & 010 & 011 & 001 & 101 & 100 & 110 \\
\cline { 2 - 8 } & 0 & 000 & 111 & 000 & 111 & 000 & 111 \\
\cline { 2 - 7 } & -1 & 001 & 101 & 100 & 110 & 010 & 011 \\
\hline
\end{tabular}


Controlador propuesto: Como se mencionó en la sección anterior, el control del par y el flujo se puede realizar mediante el fasor espacial de enlaces de flujo del estator, y este a su vez puede ser modificado mediante el fasor de voltaje del estator. Este último se puede dividir en dos componentes a lo largo de los ejes $x$ y $y$ del MR fijo al fasor espacial de enlaces de flujo del estator, como se muestra en la figura 6 . La componente $u_{s x}$ afecta directamente al módulo de enlaces de flujo del estator, por tanto el control del flujo se puede realizar por medio de esta componente; y la componente $u_{s y}$ afecta al ángulo del fasor espacial de enlaces de flujo del estator, por tanto el control del par electromagnético se puede llevar a cabo manipulando esta componente.

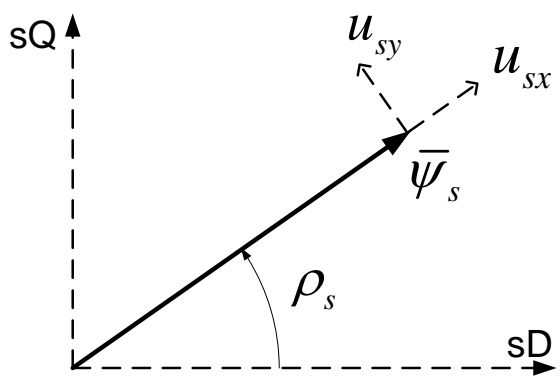

Fig. 6. Componentes del fasor de voltaje del estator.

Para realizar el control de par y flujo se puede utilizar las siguientes expresiones para las componentes del fasor espacial de voltaje del estator en el MR fijo al fasor espacial de enlaces de flujo del estator.

$$
\begin{gathered}
u_{s x}=k_{\psi} e_{\psi} \\
u_{s y}=k_{t} e_{t}
\end{gathered}
$$

donde $k_{\psi}$ y $k_{t}$ son las ganancias de los controladores de flujo y de par, respectivamente. $e_{\psi}$ y $e_{t}$ son los errores de flujo y de par. Una vez teniendo las componentes del fasor espacial de voltaje del estator en el MR fijo al fasor espacial de enlaces de flujo del estor, se pueden encontrar el fasor espacial del voltaje del estator en el MR estacionario por medio de la siguiente ecuación:

$\bar{u}_{s}=\left(u_{s x}+j u_{s y}\right) e^{j \rho_{s}}$

En la figura 7 se muestra el diagrama a bloques del controlador propuesto, el cual es muy similar al diagrama a bloques del CDP convencional (Figura 4). La principal diferencia es el modo como se calcula el fasor espacial de voltaje del estator: en el caso del CDP convencional se calcula mediante la tabla $1 \mathrm{y}$ en el controlador propuesto se calcula mediante las ecuaciones (9) y (10). Otra diferencia es el forma en la que se generan las señales de modulación del inversor $\left(S_{a}, S_{b}\right.$ y $\left.S_{c}\right)$ en el CDP convencional estas provienen directamente de la tabla 1 y en el controlador propuesto se utiliza la técnica de modulación PWM senoidal. Además en el controlador propuesto, a diferencia del CDP convencional, no se utilizan los comparadores de histéresis.

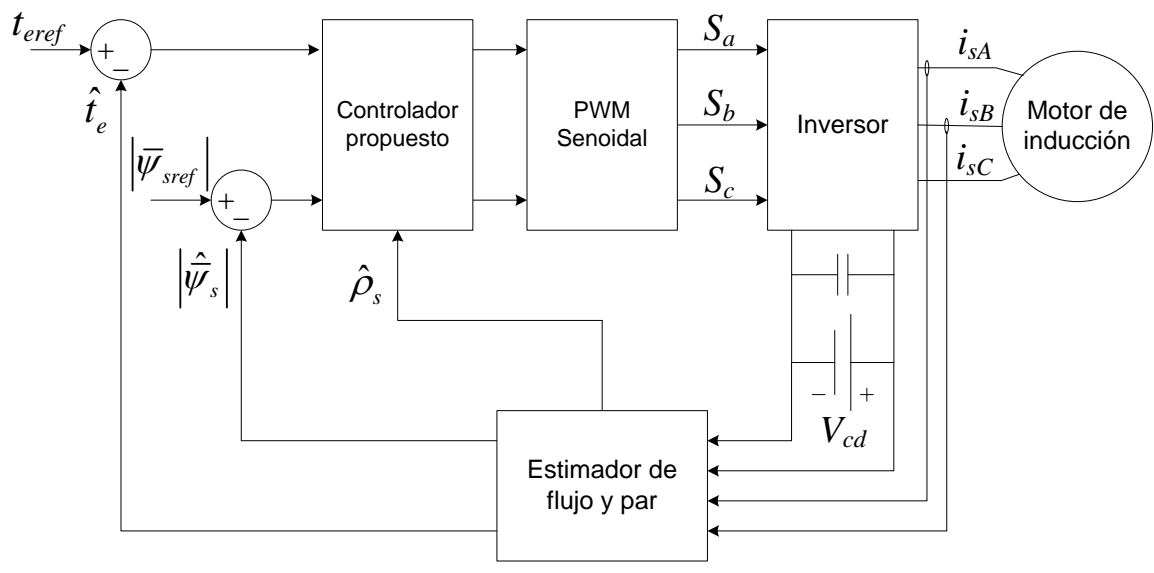

Fig. 7. Diagrama a bloques del controlador propuesto 


\section{SIMULACIONES}

En esta sección se presentan las simulaciones realizadas para evaluar el desempeño del CDP convencional y del controlador propuesto. Para ambas simulaciones: los parámetros para el $\mathrm{Ml}$ y del VE están dados en las tablas 2 y 3 ; se empleó un controlador PI para la velocidad con ganancias $k_{p}=5000$ y $k_{i}=300$; el perfil de velocidad deseada es el mismo para ambos controladores; el flujo deseado es constante e igual a 0.6 Wb-vuelta; y el voltaje del bus de cd es $300 \mathrm{~V}$.

Tabla 2: Parámetros del MI

\begin{tabular}{|l|l|}
\hline Parámetro & Valor \\
\hline Resistencia de estator, $R_{s}$ & 0.06336 \\
\hline Resistencia de rotor, $R_{r}$ & 0.073558 \\
\hline Inductancia de dispersión estator, $L_{/ s}$ & $0.8646 \mathrm{mH}$ \\
\hline Inductancia de dispersión rotor, $L_{I r}$ & $0.8646 \mathrm{mH}$ \\
\hline Inductancia magnetización estator, $L_{m s}$ & $17.913 \mathrm{mH}$ \\
\hline Inductancia magnetización rotor, $L_{m r}$ & $17.913 \mathrm{mH}$ \\
\hline Inductancia mutua estator-rotor, $L_{s r}$ & $17.913 \mathrm{mH}$ \\
\hline Momento de inercia, $J_{M I}$ & $1.0473 \mathrm{~kg} \cdot \mathrm{m}^{2}$ \\
\hline Coeficiente de fricción viscosa, $B$ & $11.5 \times 10^{-3} \mathrm{~kg} \cdot \mathrm{m}^{2} / \mathrm{s}$ \\
\hline Número de pares de polos, $n_{p}$ & 2 \\
\hline
\end{tabular}

Tabla 3: Parámetros del VE

\begin{tabular}{|l|l|}
\hline Parámetro & Valor \\
\hline Masa, $m$ & $1366 \mathrm{~kg}$ \\
\hline Coeficiente aerodinámico, $C_{d}$ & 0.23 \\
\hline Área frontal, $A$ & $2.66 \mathrm{~m}^{2}$ \\
\hline Coeficiente de fricción, $-r r$ & 0.015 \\
\hline Cociente de la transmisión, $G$ & 5.5 \\
\hline Eficiencia de la transmisión, $-g$ & 0.95 \\
\hline Radio de los neumáticos, $r$ & $0.287 \mathrm{~m}$ \\
\hline
\end{tabular}

CDP convencional: Los valores de las bandas de los comparadores de histéresis utilizados son $\Delta \psi=0.01$ Wb vuelta y $\Delta t_{e}=2.5 \mathrm{~N} \cdot \mathrm{m}$. Con estos valores se obtuvo una frecuencia de conmutación máxima de 18.2 $\mathrm{kHz}$. La velocidad deseada, así como la velocidad desarrollada por el VE en simulación se muestran en la figura 8.a, en la figura 8.b se tiene el error de velocidad el cual oscila entre $-0.028 \mathrm{~m} / \mathrm{s}$ y $0.012 \mathrm{~m} / \mathrm{s}$. La salida del controlador PI de velocidad, es decir, el par deseado, y el par estimado se aprecian en la figura 9.a. El error de par presentado en la figura 9.b no permanece todo el tiempo dentro de la banda de histéresis $\Delta t_{e}=2.5 \mathrm{~N} \cdot \mathrm{m}$ pues llega a tener valores cercanos a $3.5 \mathrm{~N} \cdot \mathrm{m}$.

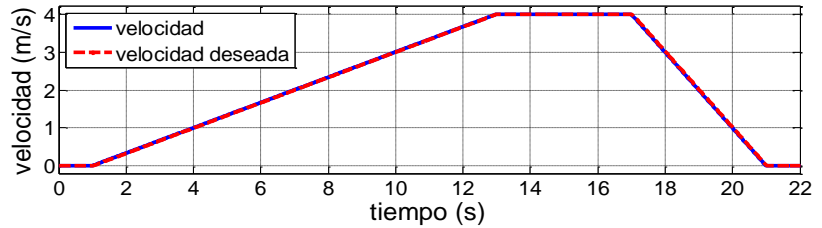

a)

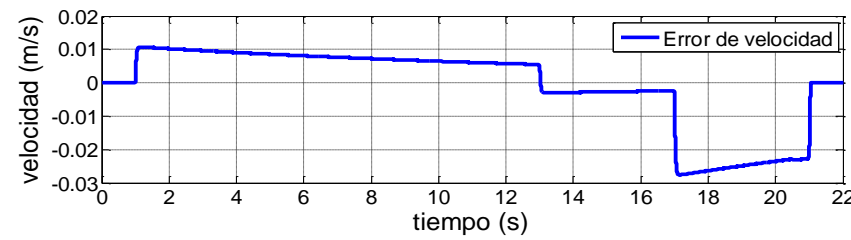

b)

Fig. 8. a) Velocidad vs. velocidad deseada y b) error de velocidad

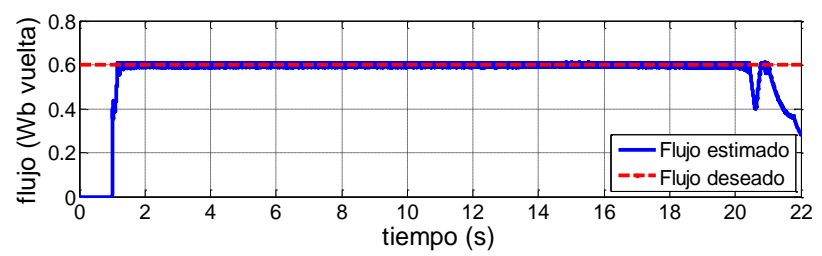

a)

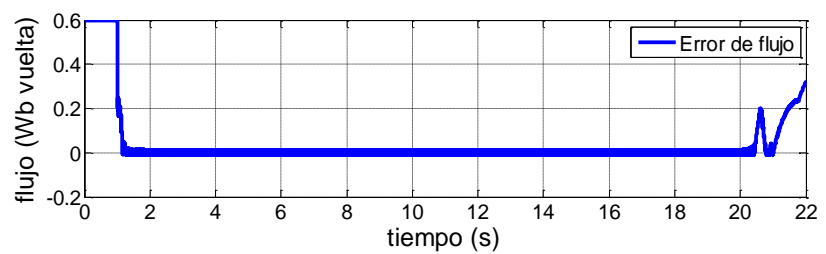

b)

Fig. 10. a) Flujo deseado vs. estimado y b) error de flujo

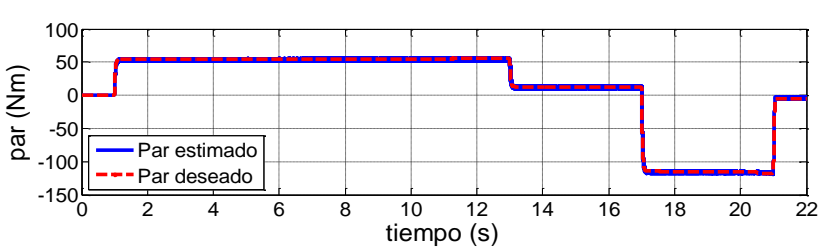

a)

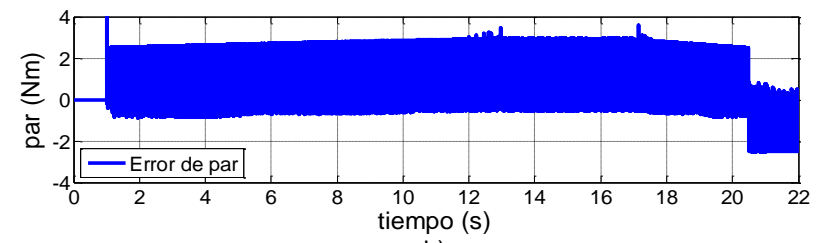

b)

Fig. 9. a) Par deseado vs. par estimado y b) error de par

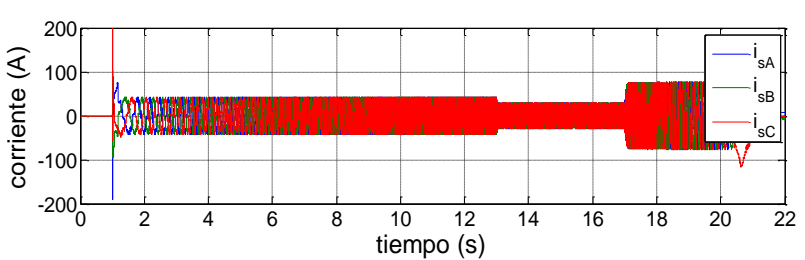

a)

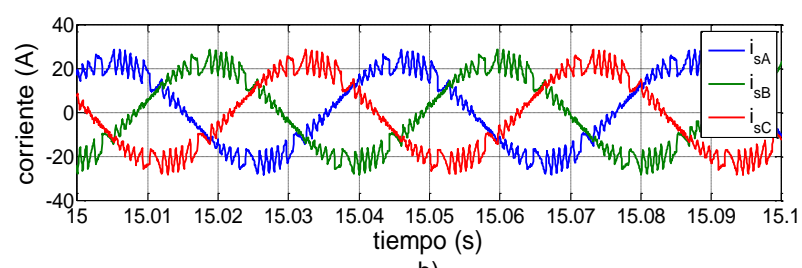

b)

Fig. 11. Corrientes del estator: a) 0 a 22s y b) $15 \mathrm{~s}$ a $15.1 \mathrm{~s}$ 
El flujo estimado tiene un buen seguimiento respecto al flujo deseado (Figura 10) durante el intervalo de tiempo $1 \mathrm{~s}<\mathrm{t}<21 \mathrm{~s}$. De 0 a $1 \mathrm{~s}$ el seguimiento de flujo no es bueno debido a que durante este tiempo el error de par es prácticamente cero y de acuerdo con la tabla 1, cuando esto sucede no importa que exista error de flujo el voltaje del inversor es cero. Algo similar sucede en el último segundo de simulación. La magnitud de las corrientes que circulan en los devanados del estator está estrechamente relacionada con el par electromagnético desarrollado, de aquí que su valor varíe a lo largo del tiempo de simulación como se muestra en la figura 11.a. Un acercamiento a las corrientes durante el intervalo de tiempo de $15 \mathrm{~s}$ a 15.1 se presenta en la figura 11.b.

Controlador propuesto: Los valores para las ganancias de los controladores de flujo y de par son $k_{\psi}=180 \mathrm{y}$ $k_{t}=1$, respectivamente. En la figura 12 se presentan la velocidad deseada vs. velocidad desarrollada por el VE, así como el error de velocidad. La velocidad deseada es la misma que para el CDP convencional, así mismo la velocidad desarrollada por el VE y el error de velocidad son muy similares a los obtenidos en la simulación del CDP convencional (Figura 8), esto se debe a que el controlador de velocidad es el mismo para ambas simulaciones. El par deseado y el par estimado se aprecian en la figura 13.a. El error de par presentado en la figura 13.b es menor al obtenido en la simulación del CDP convencional. Para el controlador propuesto el error de par no supera $1.5 \mathrm{~N} \cdot \mathrm{m}$ mientras que para el CDP convencional este error llega a ser mayor de $3 \mathrm{~N} \cdot \mathrm{m}$. Como se aprecia en la figura 14, el control de flujo también presenta mejor desempeño en el controlador propuesto que en el CDP convencional. El error de flujo, una vez pasado el transitorio, es muy pequeño, oscila entre $-1.5 \times 10^{-3} \mathrm{~Wb} \cdot \mathrm{vuetla}$ y $1.5 \times 10^{-3} \mathrm{~Wb} \cdot \mathrm{vuetla}$. Las corrientes del estator se presentan en la figura 15, su magnitud es muy similar a las obtenidas con el CDP convencional, sin embargo como se aprecia en la figura 15.b su distorsión es mucho menor en el controlador propuesto.

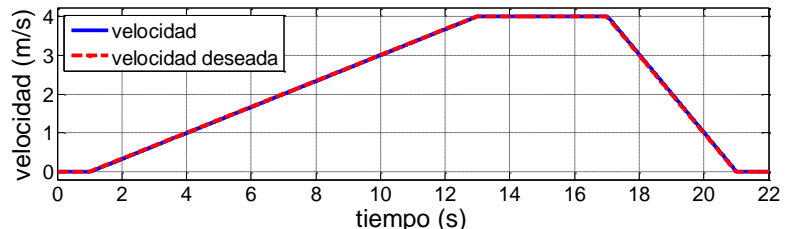

a)

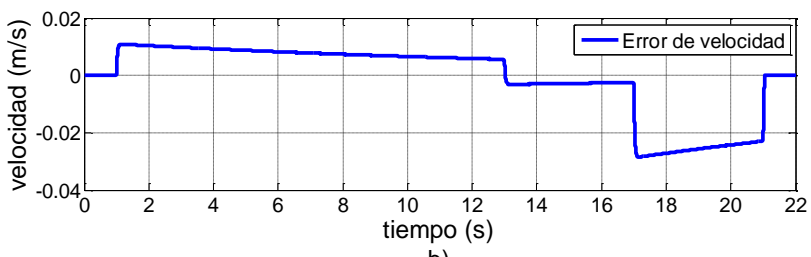

b)

Fig. 12. a) Velocidad deseada vs. velocidad y b) error de velocidad

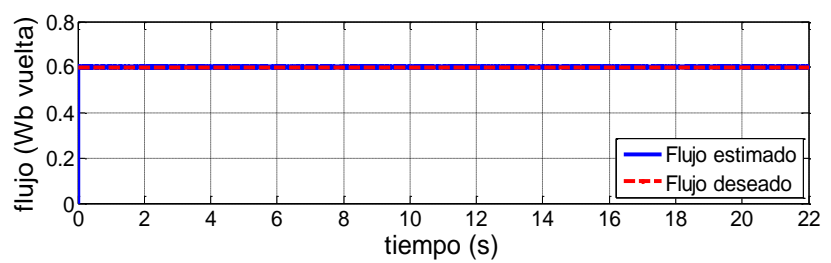

a)

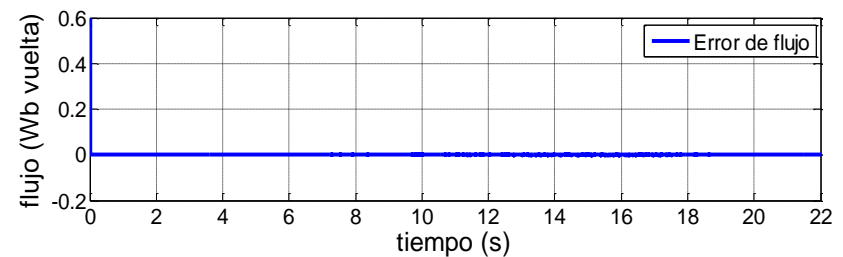

b)

Fig. 14. a) Flujo deseado vs. estimado y b) error de flujo

\section{CONCLUSIONES}

El controlador directo de par convencional representa una buena opción para realizar control de par del MI. Sin embargo presenta algunos inconvenientes: un elevado rizo de par, la frecuencia de conmutación del inversor no es fija. El valor de las bandas de histéresis juegan un papel fundamental en el desempeño del CDP convencional. Si se utilizan valores muy grandes el desempeño será pobre, ocasionando a la vez que

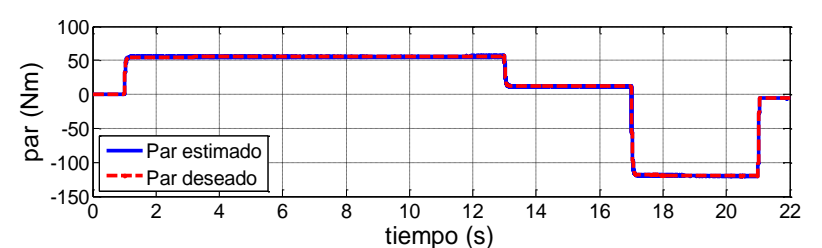

a)

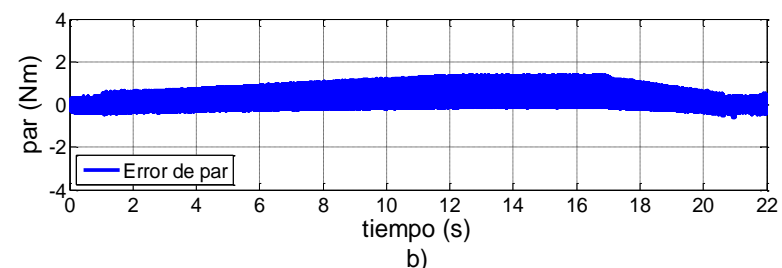

Fig. 13. a) Par deseado vs. par estimado y b) error de par

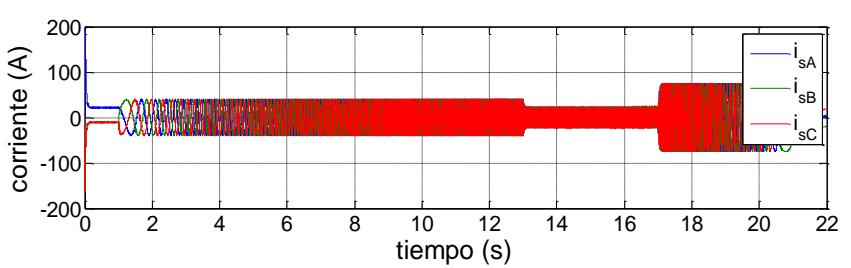

a)

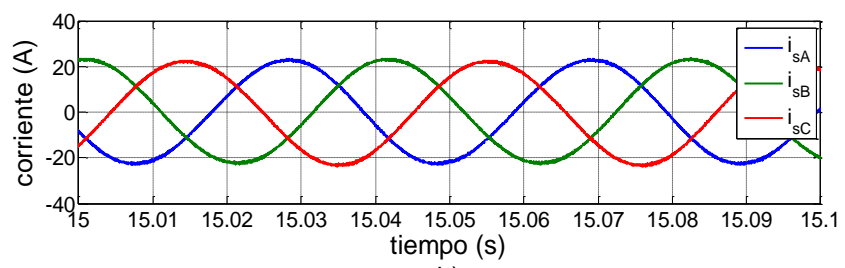

Fig. 15. Corrientes del estator: a) 0 a 22s y b) $15 \mathrm{~s}$ a $15.1 \mathrm{~s}$ 
la frecuencia de conmutación sea baja. En cambio sí utilizan valores demasiado pequeños el desempeño mejora significativamente, pero también aumenta la frecuencia de conmutación pudiendo rebasar el límite impuesto por los dispositivos semiconductores.

Con base en el principio del control directo de par, se tiene que el control del par y flujo se puede llevar a cabo mediante el fasor espacial de enlaces de flujo del estator. Considerando que su movimiento es circular, si se actúa sobre su componente "radial" se afectará su módulo, es decir, el flujo; en cambio si se actúa sobre su componente "tangencial" se modificará su ángulo y a la vez el par. Por otra parte el fasor espacial de enlaces de flujo del estator se puede modificar directamente mediante el fasor espacial de voltaje del estator. En el controlador propuesto se trabaja en el MR fijo al fasor espacial de enlaces de flujo del estator. La componente $u_{s x}$ del fasor espacial de voltaje del estator es proporcional al error de flujo, y la componente $u_{s y}$ es proporcional al error de par.

El desempeño del controlador propuesto es mejor al desempeño del CDP convencional, bajo las mismas condiciones de simulación: al tener menores errores de par y flujo, y menor distorsión en las corrientes del estator. El tener un rizo de par elevado aunque puede no ser perceptible en la velocidad del VE, sin embargo, este rizo puede afectar los componentes del VE.

\section{REFERENCIAS}

Ahmed, T. ; Das, A. ; Halder, K.K., Comparison of DTC and FOC for FSTP inverter fed IPMSM drives. 2013 International Conference on Electrical Information and Communication Technology (EICT), pp. 1-5, (2014)

Andrada, P., Torrent, M., Blanqué, B., y Perat, J.I., Accionamientos de Reluctancia Autoconmutados para Vehículos Eléctricos. Información tecnológica, 16(5), 79-87, (2005).

Depenbrock, M., Direkte Selbstregelung (DSR) fiir hochdynamische Drehfeld-antriebe mit Stromrichterschaltung. ETZ A 7, pp. 211-18, (1985).

Durán, M., Aguilera, J., Guerrero-Ramírez, G., Claudio, A., Vela, L. G., Gudiño-Lau, J., Modelado del Sistema de Tracción para un Vehículo Eléctrico. Congreso Anual 2010 de la Asociación de México de Control Automático. pp. 473 - 478, Puerto Vallarta, Jalisco, México, (2010).

Gonder, J.D., Route-Based Control of Hybrid Electric Vehicles. In SAE World Congress \& Exhibition, 2008. SAE International. (2008).

Hassankhan, E., Khaburi, D. A., DTC-SVM Scheme for Induction Motors Fed with a Three-level Inverter. World Academy of Science, Engineering and Technology, pp. 168-172, (2008)

Hegazy, O., Barrero, R., Van Mierlo, J., El Baghdad, M., Lataire, P., Coosemans, T., Control, analysis and comparison of different control strategies of electric motor for battery electric vehicles applications, 15th European Conference on Power Electronics and Applications (EPE), pp. 1-13, (2013)

Idris, N. R. N., Toh, C. L., Elbuluk, M., A New Torque and Flux Controllers for Direct Torque Control of Induction Motors. IEEE Transactions on Industry Applications, (2006).

Schouten, N.J., Salman, M.A., and Kheir, N.A., Fuzzy logic control for parallel hybrid vehicles. IEEE Transactions on Vehicular Technology, Vol. 10, pp. 460-468, (2002).

Sciarretta, A., Guzzella, L., Control of hybrid electric vehicles. Control Systems, IEEE, 27(2), pp. 60 -70, (2007).

Takahashi, Noguchi, T., A new quick-response and high efficiency control strategy of an induction motor. IEEE Trans. Ind. Appl., vol. IA-22, no. 5, pp. 820-827, (1986).

Vas, P., Sensorless Vector and Direct Torque Control. Oxford University Press, (1998).

Vasudevan, M., Arumugam, R., Simulation of Viable Torque Control Schemes of Induction Motor for Electric Vehicles. 5th Asian Control Conference, vol. 2, pp. 1377 - 1383, (2004). 